\title{
Immediate angioplasty in the United Kingdom
}

\section{The time has not yet come}

Primary percutaneous transluminal coronary angioplasty (PTCA) has recently been advocated as the treatment of choice for patients with acute myocardial infarction. ${ }^{1-4}$ Three randomised trials, one in the Netherlands and two in America, have suggested that primary PTCA might achieve higher patency rates than thrombolysis with intravenous streptokinase or tissue plasminogen activator ( $t-P A$, alteplase).$^{2-4}$ In addition one study suggested that the severity of the residual lesion was reduced by primary PTCA and that there was less impairment of left ventricular function at the time of discharge. ${ }^{3}$ Two of the studies, however, showed no improvement in left ventricular function. ${ }^{24}$

In one study there were fewer strokes in the group treated by primary PTCA, but at the expense of an increased risk of arterial damage requiring surgical repair. ${ }^{2}$ There were too few patients (only four in each category) for firm conclusions to be drawn.

The drive towards the widespread adoption of primary PTCA is based on data obtained from 537 patients who were randomised in the two studies that included end points other than left ventricular function. ${ }^{23}$ In these studies there was a significant reduction in recurrent ischaemia as judged by the occurrence of angina, unstable angina, recurrent infarction, and abnormal exercise test results. It is these findings that command the closest attention because they hint at a possible reduction in death rate particularly in high risk patients. Only the study of Grines and colleagues showed a reduction in mortality $(2.6 v 6.5 \%, P=0.06)$ and this was significant only if deaths and non-fatal reinfarctions were combined (5.1 $v 12 \cdot 0 \%, P=0.02)$. These data are impressive but perhaps not enough to cause a wholesale change in the policies adopted for the management of the commonest cause of acute medical admission in most hospitals in the developed world. Perhaps the crucial issue, when considering the introduction of primary PTCA into routine practice, is whether the results of randomised trials that have been undertaken in highly specialised, interventional centres are valid when applied to the populations of unselected patients presenting to the coronary care units of less specialised community (district general) hospitals.

\section{LOGISTICS}

In the United Kingdom, less than $10 \%$ of hospitals have facilities for cardiac catheterisation and in most cases PTCA is performed only in those centres that also undertake cardiac surgery. ${ }^{56}$ Although these surgical centres are mostly situated in densely populated areas, their catchment populations taken together account for only a small proportion of the whole population at risk.

In an average district in the United Kingdom of 250000 people with an average standardised mortality ratio for coronary heart disease, there will be about 500 cases a year of acute myocardial infarction that might be considered for primary PTCA. Although the rates for elective PTCA vary enormously from district to district, at present the average rate of activity is about 100 cases per average district of 250000 , implying a need for a fivefold increase in activity that would surely swamp even the most efficient of surgical centres. Furthermore, if this activity was to match that of the randomised trials, this cohort of acute patients would need access to the catheter laboratories at these centres within four hours of the onset of symptoms, a target that is not yet feasible even for thrombolysis in district hospitals.?

The inevitable conclusion from these arguments is that primary PTCA would need to be undertaken in district hospitals. If so, there is considerable doubt as to whether the laudable results obtained by Grines and colleagues would have been the same had their study been conducted in hospitals where facilities, staffing levels, and PTCA experience do not and are never likely to match those available in specialist centres.

The arguments outlined above may to an extent be undermined if primary PTCA was to be reserved for small subgroups of patients. It has been suggested that the procedure could be reserved for selected patients particularly those with early presentation, acute ST elevation, anterior infarction, or a contraindication to thrombolysis. $^{1}$ The data on these subgroups is not yet sufficiently strong for firm conclusions to be drawn.

\section{COST ISSUES}

It has been argued that primary PTCA might prove to be more cost effective than thrombolysis. Gibbons et al reported a lower mean hospital cost in patients treated by angioplasty compared with those receiving thrombolysis: but there were very wide standard deviations in their study of only 108 patients. ${ }^{4}$ These data are not reinforced by the twofold increase in the incidence of early coronary bypass grafting in the PTCA group reported by Grines et $a l .{ }^{2}$ In addition, there are very real pitfalls in drawing conclusions on cost implications from data derived in clinical settings very remote from the average coronary care unit in a British district general hospital. The Mayo Clinic cannot be compared with the usual health district that may or may not have a cardiac specialist. ${ }^{4}$

\section{"We are in danger of doing more and more ... for fewer and fewer patients"}

Within the United Kingdom the costing exercise would have to include the building of catheter laboratories for the $90 \%$ of hospitals that do not have them and the costs involved training staff and providing a 24 hour service. There is also considerable doubt as to whether the low complication rates reported for primary PTCA could be achieved by operators who currently undertake little or no PTCA. Complication rates have cost implications.

\section{EOUITY AND PRIORITIES}

If primary PTCA can be offered to only a few selected patients who happen to arrive at a surgical centre at a time of day when the organisation can respond within the necessary limited time frame, should the treatment be offered at all? Should place of residence and good fortune be added to the list of social factors that govern an individual's likelihood of achieving appropriate treatment-a list that includes social class, employment status, gender, race and level of health awareness? 
Secondly, we should consider whether we would be concentrating resources in the wrong area. From community studies of acute myocardial infarction, we know that mortality is highest soon after acute myocardial infarction and that despite a steady fall in hospital mortality, particularly in those patients enrolled in thrombolysis trials, the overall risk for an individual who has a heart attack has altered very little.$^{89} \mathrm{We}$ are therefore in danger of doing more and more in the way of treatment for fewer and fewer patients. Is this really the right way forward?

\section{CONCLUSIONS}

It has been suggested that primary PTCA is in danger of being "buried alive" ${ }^{10}$ An alternative view is that the pro-

1 Grech ED, Ramsdale DR. Angioplasty and acute myocardial infarction. Lancet 1994;342:191.

2 Grines CL, Browne KF, Marco J, et al. A comparison of immediate angioplasty with thrombolytic therapy for acute myocardial infarction. $N$ Engl fMed 1993.328:673-9.

3 Zijlstra F, de Boer MJ, Hoorntie JCA, Reiffers S, Reiber JHC, Suryapranata $H$. A comparison of immediate coronary angioplasty with intravenous streptokinase in acute myocardial infarction. $N$ Engl $₹$ Med intravenous strepto

4 Gibbons RJ, Holmes DR, Reeder GS, Bailey KR, Hopfenspirger MR, Gersh BJ. Immediate angioplasty compared with the administration of a thrombolytic agent followed by conservative treatment for acute myocardial infarction. N Engl F Med 1993;328:685-91.

5 A report of a working group of the British Cardiac Society: Cardiology in the cedure is in suspended animation and there it should remain until further large-scale trials guide us as to the best way forward, particularly in relation to patient selection. If primary PTCA is to be adopted into routine practice then it should be in the context of a strategy covering all opportunities for intervening from the moment symptoms begin. Such a strategy might reduce the high mortality in many patients with acute myocardial infarction more than the introduction of a single, expensive procedure for those that have already survived the period of highest risk.

R M BOYLE

Department of Cardiology, York District Hospital

district hospital. $\mathrm{Br}$ Heart $\mathcal{f}$ 1994;72:303-8.

6 Report of a working group of the British Cardiac Society: Coronary angioplasty in the United Kingdom. Br Heart f 1991;66:325-31.

7 Birkhead JS on behalf of the Joint Audit Committee of the British Cardiac Society and a Cardiology Committee of the Royal College of Physicians Society and a Cardiology Commitre

8 Fitzpatrick B, Watt GCM, Tunstall-Pedoe H. Potential impact of emergency intervention on sudden death from coronary heart disease in gency intervention on sudden death

9 Norris RM, Dixon GF, Chamberlain DA, Vincent R. Mortality from ischaemic heart disease (IHD) outside and inside hospital: the Brighton Heart Attack Study. Br Heart f 1994;71 (suppl):33P

10 Meier B. Balloon angioplasty for acute myocardial infarction: was it buried alive? Circulation 1990;82:2243-5.

\section{Experience of primary angioplasty in the United Kingdom}

\section{Spectre of an overwhelming increase in workload}

The benefits of reperfusion therapy for acute myocardial infarction are well established and most patients should now receive thrombolytic agents to achieve recanalisation of the occluded infarct-related vessel. ${ }^{1}$ However, thrombolytic therapy does have several limitations. Firstly, recanalisation is not achieved in at least $20 \%-30 \%$ of patients ${ }^{2}$ and only $50 \%$ will achieve TIMI grade 3 flow in the first 90 minutes. ${ }^{3}$ Secondly, of those patients in whom recanalisation occurs, $10 \%-30 \%$ will have early or late reocclusion (despite treatment with aspirin and heparin) leading to reinfarction, worsening left ventricular function, and death. ${ }^{45}$ Thirdly, intracranial bleeding can occur in $1 \%$ of patients, causing death in $35 \%-40 \% .^{6}$ In addition, $15 \%-20 \%$ of patients with myocardial infarction will have some contraindication to thrombolytic therapy. ${ }^{7}$

Though primary (or direct) percutaneous transluminal coronary angioplasty (PTCA) offers an opportunity to overcome most of these problems, major issues need to be addressed before this form of treatment can be applied nationwide.

Direct PTCA has been shown to have a high primary success rate (90\%-99\%) with few procedural complications and a low in-hospital mortality. ${ }^{89}$ It can establish TIMI grade 3 flow in up to $95 \%$ of patients within two hours of hospital admission ${ }^{10}$ and this has been our experience. Thrombolytic agents cannot achieve this. Primary angioplasty has a more rapid action and greater success because it can dislodge and mechanically disrupt thrombus as well as reduce any residual coronary stenosis caused by atheroma. These actions reduce the risks of recurrent ischaemia, reocclusion, and reinfarction. ${ }^{11}$ Primary PTCA also avoids the significant bleeding risks associated with systemically administered thrombolytics and reduces the formation of a haemorrhagic myocardial infarction. ${ }^{12}$ If necessary, residual intracoronary thrombus seen angiographically can be simultaneously, effectively, and more reliably treated with small doses of clot-specific thrombolytic drugs administered directly down the relevant vessel.

Primary PTCA leads to a reduction of infarct size and improved left ventricular function when compared with streptokinase therapy, with the most pronounced benefits being in those with anterior myocardial infarction presenting within two hours after the onset of symptoms. ${ }^{13}$ These impressive results are presumably the result of a rapid and effective restoration of blood flow to jeopardised myocardium. In addition to reducing the likelihood of recurrent myocardial ischaemia and reinfarction, immediate PTCA reduces the need for early additional revascularisation procedures and reduces in-hospital mortality, compared with intravenous thrombolytic therapy. Again these effects are a consequence of improved coronary blood flow and better left ventricular function. ${ }^{13}$ Brodie et al showed that after primary PTCA, patency of the infarct-related artery is the most important determinant of hospital survival and that left ventricular function measured after recovery is the most important determinant of late cardiac survival. ${ }^{14}$ The beneficial effects of primary PTCA were sustained (up to 36 months) after discharge ${ }^{1315}$ although as many as $28 \%$ of patients require a repeat PTCA for restenosis.

Primary PTCA can also be used successfully when thrombolytic therapy is contraindicated by a high risk of bleeding or an adverse haemodynamic state such as cardiogenic shock. ${ }^{1617-19}$ Indeed, it is probably the best treatment for achieving rapid reperfusion in such high risk subsets. ${ }^{19}$ 